\section{Short-term memory with different organizations of prose paragraphs*}

\author{
BARBARA S. MUSGRAVE \\ Smith College, Northamptor, Mass. 01060
}

Proactive inhibition and release phenomena were explored using four-sentence paragraphs with each sentence containing a key word for recall after a rehearsal-interference task. Each paragraph described a particular member of a class of items on each of four semantically unrelated attributes. Repetition of paragraphs describing items on the same attributes at first produced slight improvement and then decrement. Shifts to paragraphs using different attributes resulted in somewhat better recall, which again improved over early trials with repetition of the changed materials, and then declined. Findings of facilation were discussed in terms of organization learning, decrements in terms of proactive inhibition.

This study brings together two areas of research on verbal learning: studies of the generation and release of proactive inhibition (PI) and studies concerning the effects of organizational factors on learning from prose passages. The PI studies have focused on short-term memory phenomena, the prose studies on long-term memory and encoding processes. They differ in procedure, but share an important feature of the materials, namely, repetition of stimulus categories.

In studies of prose organization, an entire passage is presented during a study period, and then amsunt of learning is tested by recall of all items of information that have been presented. In this sort of study, blocked repetition of similar items aids recall.

In PI studies, only part of the study material is presented on each trial, and, as the trials proceed with similar stimuli, amount correctly recalled decreases, only to increase again if a shift is made to dissimilar stimuli.

Thus, in one case, blocked repetition improves recall; in the other, it acts as an inhibitor. However, the materials for PI studies have been considerably simpler than those for prose-organization studies. Therefore, there is the possibility that the contradiction in findings is because PI is not generated with complex materials in which the repetitiousness occurs over sets of sentences. The present study used prose materials with a PI shift-release procedure in an effort to find out whether PI does, in fact, occur with these materials.

The PI study that speaks most directly here is one by Schuberth, Lively, \& Reutener (in press), in which Ss were given sentences containing

* Thanks are due Mrs. Marilyn Ingelus for data collection. nouns from three different categories, a rehearsal-interference task, and then a recall test. The control condition continued to have sentences using nouns from the same categories, while the experimental condition, after experiencing a number of such sentences, was shifted to sentences identical in sentence frame but containing nouns from other categories. Retention of nouns decreased during trials on which the categories remained the same, thus exhibiting PI, and increased on the shift trials when the categories were changed, thus exhibiting $\mathrm{PI}$ release. This study extended the findings of previous PI shift studies (for example, Wickens \& Clark, 1968) in the following ways: (1) The stimulus for each trial consisted of a sentence instead of several discrete items, such as three semantically related words; and (2) the stimulus sentence for each trial contained words from three different categories, with repetition of the same category occurring over several sentences but not within the stimulus for any one trial.

Prose study materials that are relevant here typically present names to be associated with values on a set of attributes. For example, Frase (1969) used materials describing each of six chessmen on each of eight attributes. The materials can thus be schematized as a 6 by 8 , Names by Attributes, matrix array (Musgrave \& Cohen, 1971). Each sentence in the prose study passage coupled a name with an attribute value. The 48 resultant sentences were presented in three alternative ways: (1) blocked by name, (2) blocked by attribute, or (3) presented randomly. Recall of materials in the first two conditions was significantly better than recall in the third condition. In a serial position curve analysis, Frase reports a primacy effect but no recency effect.
By using materials like those of the prose-organization experiments, the present PI study made the following further extensions from the Schuberth et al experiment: (1) four different categories were used instead of three, and $(2)$ the four key words for recall were not presented together in one sentence, but instead in separate sentences with the four sentences presented as a prose paragraph.

The main question at issue was whether the proactive inhibition and release phenomena were robust enough to occur with this kind of prose. If so, the data might speak to "boundary conditions" for how "well organized" (blocked) prose should be for maximum ease of learning, and also to the puzzle of why such prose, unlike discrete word lists, shows primacy effects but no recency effects under free recall instructions.

\section{METHOD}

The Ss were 42 paid volunteer women, all enrolled at Smith College, who were naive to verbal learning experiments and whose native language was English. On appearance at the laboratory, each $\mathbf{S}$ was assigned to one of the three conditions, which were prescheduled to follow each other consecutively for each triple of Ss.

The materials described two sets of six college men each, with each man described on each of four descriptive dimensions-thus a 12 by 4 matrix. The two sets differed completely from each other in what the categories of the four descriptors were. The six men of one set were each described as playing a particular musical instrument, having a pet dog of a particular species, driving a particular make of car, and subscribing to a particular magazine. The descriptor categories of the other set of six men were: a favorite shellfish, a Latin American country visited during the summer, a game he enjoyed, and a father engaged in a particular profession. In each description, the instances of the four categories appeared in the exact order given above. The initial letters of the four key descriptive words in a paragraph did not alliterate or spell a word. No instance was ever repeated.

The study and test materials were presented in 12 small booklets, one for each trial. Following the cover sheet. the first page presented a stimulus paragraph describing one of the men. The $\mathrm{S}$ was asked to read the paragrapt. aloud, and $E$ recorded the time taken to do so. The $S$ then turned immediately to the second page on which was typed a series of random $\mathrm{numbers}$. As a 15 .sec rehearsal-interference task, $S$ was asked to bracket and add each 
Trials

\begin{tabular}{|c|c|c|c|c|c|c|c|c|c|c|c|c|}
\hline \multirow[b]{2}{*}{ Condition } & & & & & & & & & & & \\
\hline & 1 & 2 & 3 & 4 & 5 & 6 & 7 & 8 & 9 & 10 & 11 & 12 \\
\hline Condition I & 6.29 & 7.29 & 6.57 & 6.64 & 6.86 & $6.71^{*}$ & 7.71 & 6.93 & 6.93 & 6.71 & 6.07 & $6.64^{*}$ \\
\hline Control & 6.42 & 6.42 & 6.71 & 3.79 & 5.86 & 5.79 & $6.50^{*}$ & 6.93 & 6.93 & 6.79 & 5.50 & 6.64 \\
\hline Alternating & 7.21 & $6.79 *$ & $6.00^{*}$ & $5.64^{*}$ & $6.42^{*}$ & $5.57 *$ & $5.57^{*}$ & $5.50 *$ & $6.64^{*}$ & $5.64^{*}$ & $6.29^{*}$ & $4.93^{*}$ \\
\hline
\end{tabular}

* Shift trial

successive pair of numbers and record each sum beneath the pair. The $S$ then turned to the third page, which had four underlined blanks, and attempted for up to $20 \mathrm{sec}$ to write the four key descriptive words. The $\mathrm{S}$ s were urged to write these in the order presented in the paragraph, but if this proved impossible, to recall them in any order. Following the completion of one booklet, $S$ turned immediately to the next, and so on through the 12 trials.

The same materials were used in all conditions, with complete counterbalancing for particular materials within each condition. Condition I presented five men described with one set of four categories; then six men described on the other set: then, for the last trial, the sixth man of the first set. The control condition presented men described on one set of categories for the first 6 trials and men described on the other set for the last 6 . Thus, a comparison could be made between shiiting on the 6 th trial to a new set of categories (Condition I) and continuing on that trial with the set previously presented (control). Similarly, Condition I shifted on the 12 th trial and the control did not. The particular items presented on the 6 th and 12 th trials were counterbalanced between sets of categories within conditions and were identical across conditions.

The third condition (alternating) did not block the descriptors using the same categories. Instead, each trial was a shift from one set of four categories to the other set.

Table 2

Means and SDs for Total Number Correct Responses Over Three-Trial Blocks from the Two Category Sets for the Shift Conditions

\begin{tabular}{|c|c|c|c|}
\hline \multirow[b]{2}{*}{ Condition } & \multicolumn{3}{|c|}{ Three-Trial Blocks } \\
\hline & $\begin{array}{c}\text { Last } \\
\text { Three/ } \\
\text { First } \\
\text { Set }\end{array}$ & $\begin{array}{c}\text { First } \\
\text { Three/ } \\
\text { Second } \\
\text { Set }\end{array}$ & $\begin{array}{c}\text { Last } \\
\text { Three/ } \\
\text { Second } \\
\text { Set }\end{array}$ \\
\hline \multicolumn{4}{|l|}{ Condition I } \\
\hline Mean & 20.21 & 21.36 & 19.71 \\
\hline SD & 3.38 & 2.27 & 3.58 \\
\hline \multicolumn{4}{|l|}{ Control } \\
\hline Mean & 16.41 & 20.36 & 18.93 \\
\hline SD & 3.70 & 4.60 & 5.01 \\
\hline
\end{tabular}

\section{RESULTS ${ }^{1}$}

Each of the four possible responses on each trial was scored 1 point if it was the correct word and an additional point if it was written in the blank representing its order of presentation in the study paragraph. Thus, a maximum score was 8 points per trial

As can be seen in Table 1. Condition I scores fell from Trial 5 to Trial 6, the shift trial. The control condition increased correct responding on its shift trial from the sixth to the seventh. And performance on the shifted sixth trial for Condition I exceeded that of the nonshifted sixth trial for the control condition. None of these comparisons concerning single trials showed reliable differences.

To make the data more comparable to those of other PI shift studies that have grouped triplets of items of one category as a trial, and to the study by Schuberth et al, Table 2 presents for Condition I and the control condition the mean total scores for the last three trials of the first set of categories (Last Three/First Set), and for the first three trials of the second set (First Three/Second Set) and the last three trials of the second set (Last Three/Second Set). The results were in accord with other shift studies in showing improvement at the time of shift to the second set of categories and then impairment as trials continued to the second set. A 2 by 3 mixed design Anova indicated no significant differences between conditions, $F(1,26)=2.36$; but the differences between blocks of trials and the interaction of Conditions by Blocks of Trials were reliable, $\mathrm{F}(2,52)$ $=9.33, \mathrm{p}<.01$, and $F(2.52)=4.36$. $\mathrm{p}<.05$, respectively. A Newman-Keuls test showed that these significant findings rested exclusively on differences between the control condition's poor performance on Last Three/First Set and all other mean values.

A comparison on the first set between the first and last block of three trials could be made only for the control condition, as it alone was presented six consecutive trials on the first set. Performance fell from a mean of 19.57 for the First Three block to 16.41 for the Last Three block, with only one $\mathrm{S}$ showing a reversal. This difference was significant beyond the
01 level according to a Wilcoxon test.

Of interest concerning Ss, organization of the task is a comparison of performance on the first set of categories presented vs the second set, as shown in Table 3 . Where study materials were blocked (Condition I and control), there was improvement for the second set. But in the alternating condition, the set of categories presented first (that is, on the first, third, fifth, etc., trials) elicited better performance than the set presented second (second, fourth, sixth, etc., trials). A 3 by 2 mixed design Anova resulted in only one significant finding: the interaction of Conditions by First or Second Set, $F(2,39)=8.78, p<.01 . A$ Newman-Keuls test showed that the performance of Ss on the second set in the alternating condition was reliably poorer than performance on the first set. Also, it was reliably poorer than performance on the second set of the Ss in Condition $I$ and in the control condition. Performance on the first set for the contro! condition was also significantly lower than performance with either of the two sets in Condition I, but not with its own second set.

\section{DISCUSSION}

One way of interpreting these findings is that repetition of categories across trials can produce both proactive facilitation and inhibition effects on short-term recall of this sort of material.

As to facilitation. trial-by-trial inspection of the results for Condition I and the control condition reveals that performance improved over the first few trials on a particular

Table 3

Means and SDs ior Total Number Correct Responses by Presentation Order of Category Sets

\begin{tabular}{lrr}
\hline & \multicolumn{2}{c}{ Presentation Order } \\
\cline { 2 - 3 } Condition & \multicolumn{1}{c}{ First } & Second \\
\hline Condition I & & \\
$\quad$ Mean & 40.28 & 41.07 \\
$\quad$ SD & 5.70 & 4.81 \\
Control & & \\
$\quad$ Hean & 35.71 & 39.28 \\
SD & 7.58 & 9.24 \\
Alternating & & \\
Mean & 38.14 & 34.07 \\
SD & 7.19 & 8.02 \\
\hline
\end{tabular}


set of categories. This improvement cannot be attributed to ease or difficulty of particular paragraphs, as paragraphs had been counterbalanced within conditions by trial. From postexperimental discussion, one gleans that $S$ s were using the categories as an organizational framework, probably both for perceptual input and memorial output, and needed more than one trial to discover and learn what the categories were.

However, the advantages of categorical stability were apparently offset by at least some buildup of PI as the repetition proceeded, for the early trials showing improvement were followed by a sharp fall in recall. Thereafter, throughout the remainder of the repetitive trials, except for the second set in Condition I, there was some improvement, but performance did not return to the highest level of the beginning trials.

Turning from trial-by-trial inspection to results with data grouped across three-trial blocks, there is again evidence of the inhibitory effects of repetition, particularly in the significant decline in the control condition from the first to the second block on the first set. That there was improvement for both shift conditions following the shift (significant improvement for the control condition) is also, in a backhanded way, evidence of the deleterious effects of repetition.

The release of inhibition accompanying the shift was not as great as that exhibited in studies using within-trial repetition of a single category or as great as Schuberth et al found with stimuli containing instances of three different categories repeated across trials. The smaller shift difference here mav be due either to better performance on the last block of trials preceding the shift or to poorer performance on the first block following the shift, or both. In relation to the first possibility, the PI decrements might have been greater had the lists been longer. Schuberth's study used nine items from each category, whereas six was the largest number of repetitions used here. As to the second possibility, the use of four different categories per stimulus, instead of the previous single category or at most three, makes the first postshift trial difficult as well as releasing, thus depressing the totals for the first postshift block of trials.

The alternating condition demanded that Ss switch from one set of categories on one trial to the second set on the next, and then back to the first, and so on. In general, performance worsened throughout the 12 trials. Perhaps the difficulty of constantly shifting from one set of categories to the other offset the facilitating effects of knowing what the categories were, while permitting the proactively inhibiting effects of this knowledge to accumulate.

The interaction of Conditions by Order of Presentations of the Category Sets is of interest. The two blocked conditions resulted in better performance on the set presented second, exhibiting something of a learning-to-learn positive transfer effect. On the contrary, in the alternating condition, the second set produced significantly poorer performance than the first set and also than the second set of the other two conditions.

This experiment indicates that Ss are sensitive to organizational patterning extending across paragraphs throughout a total prose passage. The decrements in short-term recall found here with as few as six repetitions can be coupled with Frase's finding of a primacy effect when Ss were reading an entire passage and then trying to recall the attributes of six concepts presented in a repetitious organizational structure. Although what has been called "well-organized" text has resulted in better acquisition than sentences sequenced at random, and here Condition I and the control condition nonsignificantly exceeded the alternating condition in total recall scores, there may be both an upper as well as a lower bound to the number of items that should be presented in such a systematic way. Perhaps even variety in the ordering of the descriptive categories would serve to dispel the inhibition arising from too great a repetitiousness.

\section{REFERENCES}

FRASE, L. T. Paragraph organization of written materials: The influence of conceptual clustering upon the level and organization of recall. Journal of Educational Psychology, 1969, 60 . 394-401.

MUSGRAVE, B. S., \& COHEN, J, C. Relationships between prose and list learning. In E. Z. Rothk opf and P. E. Johnson (Eds.), lerbal learning research and the technology of written instruction. New York: Teachers College Press, 1971.

SCHUBERTH, R. E., LIVELY, B. L., \& REUTENER, D. B. Release from proactive inhibition in the recall of sentences. Journal of Experimental Psychology, in press.

WICKENS, D. D., \& CLARK, S. E. Osgood dimensions as an encoding class in shorteterm memory. Journal of Experimental Psychology, 1968. 78, $580-584$. 